\title{
CONGRESS ABSTRACTS
}

SPL-1

\author{
THE $\mathrm{CO}_{2}$ LASER IN THE TREATMENT OF VASCULAR LESIONS \\ Isaac Kaplan MD PhD \\ Professor, Consultant Plastic \& Maxillofacial Surgeon, Israel
}

The advantage of the $\mathrm{CO}_{2}$ Laser over all other lasers in the treatment of vascular lesions such as haemorrhoids, cavernous haemangiomata, teleangiectasia, and others will be demonstrated.

SPL-2

\section{HISTORY OF IPTA \\ Toshio Ohshiro MD PhD}

Japan Medical Laser Laboratory, Shinanomachi, Tokyo

In the late 1960's, Endre Mester, our Godfather of Laser Therapy, opened a new genre of laser therapy where a non-thermal tissue reaction was used to treat severe ulcers. That led to the development of a dedicated therapy for wound healing and for pain attenuation. In 1988, I authored a book on laser therapy, "LOW LEVEL LASER THERAPY, A PRACTICAL INTRODUCTION" which was published by the famous medicoscientific publishing house of John Willey\& Sons Ltd., Chichester, UK. They asked me to be the Editor-in-Chief "Laser Therapy", a journal for LLLT. As the Founding-Editor, I exhibited the journal on the occasion of the joint Congress of European Medical Laser Association (EMLA), International Nd: YAG Laser Society, and the British Medical Laser Association (BMLA). One thousand of the copies were distributed to attendees in the first day of the congress. And so, John Willey \& Sons proposed that we should organize the International Laser Therapy Association: ILTA. I was elected President-elect of the new ILTA and we held the first congress of ILTA in Okinawa in 1990. The second ILTA congress was held in London under the presidency of Prof. Mary Dyson, the third ILTA congress was held in Barcelona under the presidency of Prof. Mario Trelles. Prof. Trelles suggested that we should make a new association, the World Association of Laser Therapy: WALT by merging ILTA with International Society for Low Power Laser Application in Medicine: ISLPLAM and the proposition was accepted. The first WALT congress was held in Israel in 1996 under the presidency of Prof. Shimon Rochkind, the second WALT congress was in Kansas City under the presidency of Prof. Chukuka Enwemeka, the third WALT congress was in Athens under the presidency of Prof. Nic Nicolopoulos, the forth was in Tsukuba city, Japan, under the presidency of Prof. Kazuo Hanaoka, and the fifth WALT congress was in Saõ Paulo in 2004 under the presidency of Prof. Aldo Brugnera. Before the fifth WALT, it was decided to merge Laser Therapy with a Mary Ann Liebert journal to form the Journal of Photomedicine and Laser Surgery. The Editor-in-Chief of Laser Therapy at that time, Prof. Chukuka Enwemeka, had caused suspension of the publication of Laser therapy for more than 2 years, and this is what led to the merger. However, international needs continue to grow in the field of phototherapy, pure LLLT and photodynamic therapy with low incident levels of laser and light energy. We therefore published a pure LLLT and Phototherapy journal in 2004 and "Laser Therapy" was alive again. A new association was formed to meet the same needs as the journal, and the International Phototherapy Association (IPTA) was officially formed in 2005. Our republished journal "Laser Therapy" was accepted as the official journal of IPTA, and the first IPTA Congress will be held on July 1st and 2nd in Suwa City, Japan, under the presidency of Prof. Yoshimi Asagai. 
SPL-3

\author{
THE HISTORY OF CLINICAL PDT \\ Tetsuya Okunaka, Harubumi Kato*
}

Respiratory disease center, Sanno Hospital, International University of Health and Welfare, *Department of Surgery, Tokyo Medical University

Chemical sensitization of live tissues by light was first reported in 1900 by Oscar Raab and his supervisor von Tappeiner. They applied acridine on human tumors, on lupus lesions and condylomata of female genitalia and treated the lesions successfully with light. In 1961, Lipson demonstrated enhanced tumor fluorescence by intravenous injection of hematopophyrin derivative prepared from hemoglobin. Independently, Dougherty and his group at Roswell Park Memorial Institute began their laboratory experiment and in 1978, he reported the complete or partial response in 111 of 113 skin cancer with PDT. In 1980, Hayata and Kato treated the first case of early stage bronchogenic cancer in the world and achieved CR. Since then more clinicians began to examine the possible role of PDT in treatment of cancer with a view toward eradication of early disease and palliation of more advanced tumors such as gastric, esophageal, cervical, bladder, skin, ENT cancers and brain tumor were introduced. In Japan, PDT for early lung, gastric and esophageal cancers were received government approval on October 1994. In recent topics of PDT were treatment for non-neoplastic diseases including acne, age-related macular degeneration. In this paper, the history of PDT for non-neoplastic disease as well as malignant tumors will be presented.

SPL-4

\title{
BRIDGING BY BIO-MEDICAL ENGINEERING FROM MODERN MEDICINE TO TRADITIONAL MEDICINE Kazuhiko Atsumi , MD,Ph D.
} Prof, Emeritus, Tokyo University

Modern Western medicine, namely conventional medicine, is based on science and has been contributed to cure the desperate patients in the World. However, according to the reductionism of science it has lost the way to understand the patients with holistic view. It seems to cause recently some confusion in the medical \& health care services, in particular, in the developed country. On the other hand, traditional medicine and complementary \& alternative medicine (CAM) such as acupuncture, message, herb, yoga etc have been contributing to prevent diseases and promote health. These CAMsl are considered not to be approved scientifically on safety \& efficacy, therefore, the scientific evaluation and evidence on CAMs are required . The scientific methodologies such as biomedical engineering will be necessary for the bridging between MWM and CAMs. Laser technology and medicine will be one of the most important key tool in the application of biomedical engineering for bridging.

PRL-1

15 YEARS' EXPERIENCE OF LLLT FOR CHILDREN WITH SPASTIC CEREBRAL PALSY Yoshimi Asagai

Shinano Handicapped Children's Hospital

Previous reports on therapy of intensive functional training for treatment of cerebral palsy have not been supported by confirmed efficacy, so they have the therapy has not been generally applied. For this reason, no reliable methods for suppressing myotonia are available, and the intensive functional training is considered a contraindication for treating spastic type cerebral palsy because it might aggravate the myotonia in some patients. In 1991, we introduced LLLT as a new therapeutic method for suppressing myotonia in cerebral palsy, and reported that it was useful as a supplementary treatment that enhanced the efficacy of functional training, being evidenced by the reliable myotonia suppression effect. The method has been applied to 2,000 children with cerebral palsy. We assessedsuccessively the changes in gross movement by hospital treatment with intensive functional training for $1 \square ` 2$ months according to the objective assessment criteria, 
gross motor function measure (GMFM), which is commonly accepted as the global standard. The severity of the disease was classified according to the gross motor function classification system (GMFCS). When the development of motor function was compared separately by disease severity with the cross-sectional motor growth curve, in the cases of the GMFCS level III a significant improvement was observed in patients of up to around age 8 . A significant improvement was observed inpatients of up to around 8 years old, especially up to 3 years old, when compared with the cross-sectional motor growth curve, even if the GMFCS level was IV. When compared with patients at other training sites, where only functional training therapy was applied without LLLT, the efficacy of functional training was clearly enhanced by combination with LLLT.

WS-1-1

\section{HOW TO TREAT SPASTIC CEREBRAL PALSY WITH LLLT \\ Yoshimi Asagai \\ Shinano Handicapped Children's Hospital}

The laser used was a GaAlAs diode laser, giving output powers in continuous wave of $100 \mathrm{~mW}$, at wavelength of $810 \mathrm{~nm}$. The laser was applied to the target points in contact method for 30 seconds per point. Energy densities were $288.3 \mathrm{~J} / \mathrm{cm} 2$. The points selected corresponded to the standard points used for acupuncture or nerve block, and treatment points were also selected on the bellies of the major spastic based on the degree of tension and tonic spasms present in those muscles. For the suppression of spasm in the neck and trunk, the laser was applied for 2-3s on successive points on the right and left paravertebral muscle from the cervical to the lumbar vertebrae, corresponding to Ohshiro's sweep method, or from the center to the periphery at approximately $4 \mathrm{~cm}$ intervals, using Ohshiro's proximal priority method.. Muscle spasms of the upper extremities were treated by applying LLLT to the points over the stellate ganglion, the suprascapular nerve, $\mathrm{m}$. pronator teres and the gohkoku acupuncture points, situated between the bases of the first and second metacarpal bones. For lower extremity spasm, LLLT was applied on points over the adductor muscles, the inpoh acupuncture point (between $\mathrm{m}$. vastus medialis and $\mathrm{m}$. sartorius, and the medial condyle of the femur), medial hamstring tendons, tibial nerve and the Achilles tendon. In addition to the temporary suppression effect of LLLT on myotonia, it is useful as a supplementary treatment in functional training for cerebral palsy. The laser irradiation method is simple, and the myotonic suppression of any sites on the body is possible within a short time

WS-1-2

\section{PROXIMAL PRIORITY TREATMENT METHOD IN LLLT Toshio Ohshiro \\ Japan Medical Laser Laboratory, Shinanomachi, Tokyo, Japan}

LLLT is the acronym of Low reactive Level Laser Therapy. We can say that LLLT is medical laser therapy with photobioactivation. On the other hand,: HLLT is the acronym of High reactive Level Laser Treatment or surgical laser treatment with photobiodestruction. Classification of the treatment to achieve pain attenuation is divided into 4 categories; point therapy, lineal therapy, aerial therapy and three dimensional therapy. In today's Hands-on Session, I will demonstrate my Proximal Priority Laser Therapy (PPLT), in this PPLT, we start to treat the proximal parts then move towards the peripheral parts. The living body consists of nerves, vessels and lymph channels anatomically. The center of the nerves is the head and brain, the center of the blood circulatory system is the heart and the lymph system circulates lymph by movement of the muscles. The heart beats by the order of the head while the cerebral nerves receive oxygen and nutrition from blood ejected by the heart and orders from the head are passed through the nervous systems. Both of these are essential for the activities of the living body. By orders from the head, we form hormones and enzymes which are delivered to the whole body through the blood circulation system. The lymph system is an important system which plays a major role in the control of the body's immunological function. The nerves, vessels and lymph exist in good order at every part of the body. If one of them loses function, the three of them become sluggish and trigger chronic diseases. My method is effective especially for chronic diseases. It consists of three steps; first, I irradiate the laser at the 
neck while stretching the muscles in the area of chronic pain, and then the cerebral blood flow increases. At this step, the pain is diminished by $40 \%$ to $60 \%$. As these reactions are similar to reactions of the sympathetic ganglionic block of the neck, some think that my method is the same method as the treatment of the sympathetic ganglionic block by laser. But in fact, they are not the same because we never find the enlargement of pupils with my method unlike the treatment of the sympathetic ganglionic block by laser. After a short explanation of the method, I would like to show you the PPLT by using a GaAlAs semiconductor laser system for LLLT.

\section{S-1-1}

BASIC APPROACH FOR THE MECHANISM OF EFFECT OF LOW-LEVEL LASER
IRRADIATIN ON SYMPATHETIC NERVOUS SYSTEM AND MICROCIRCURATION Toyoshi Hosokawa ${ }^{1}$, Misako Ohmori², Yasuko Kawabata? ${ }^{\text {? }}$

1. Kyoto Prefectural University of Medicine; 2. Soseikai General Hospital; and 3. Nagahama Red Cross Hospital

The clinical efficacy of low-level laser irradiation (LLLI) is widely accepted in various fields recently and there has been an increase in its use for the treatment of acute pain. Proposed mechanism of these effects are suppression of sympathetic nervous system activity, and increase in blood flow. We are convinced that LLLI has the clinical effects of treatment for pain from our research result, showed here such as the suppress of sympathetic nerves due to para-stellate ganglion laser irradiation and the potent dilation in the laser irradiated arteriole which led to marked increase in the arteriolar blood flow. (1)LLLI reduced significantly sympathetic nerves function of the patients who are suffering from various pains on face or at upper extremity supposing with highly irritated sympathetic nervous function. There was no significant change in the volunteer group with no pain. (2) The physiological basis of LLLI, was investigated with special reference to microcirculation. Male Wister rats were used. The low-level- laser was irradiated on the mesenteric arteriole about 20qm in a diameter for five minutes and red cell velocity, microvessel diameter and volumetric blood flow $(\mathrm{Q})$ were measured at pre and post laser irradiation. $\mathrm{Q}$ rapidly increased in $1 \mathrm{~min}$. after laser irradiation and slowly increased for 5 to $20 \mathrm{~min}$ until it reached plateau by $30 \mathrm{~min}$. These changes were not effected by denervation, but superfusion of $15 \mathrm{q} \mathrm{M}$ L-NAME suppressed the increase of $\mathrm{Q} \square @$ at early phase. The results suggest the involvement of nitric oxide $\square @(\mathrm{NO})$ in the increase of Q by low-level laser irradiation

\section{S-1-2}

\section{BASIC STUDIES ON PHOTO-DYNAMIC THERAPY FOR NEURONAL DISORDERS IN THE CENTRAL NERVOUS SYSTEM \\ Yousuke Kataoka}

\section{Department of Physiology, Osaka City University Graduate School of Medicine}

Although various photo-dynamic techniques are used in many clinics, the techniques for neurological disorders in the central nervous system have not been fully developed. In this paper, basic researches for manipulation of brain functions will be introduced; low-reactive level laser irradiation techniques and photo-dynamic tissue oxidation techniques. Using low-reactive level laser irradiation with a near-infrared diode laser (continuous wave; wavelength, $830 \mathrm{~nm}$; power density, $1-5 \mathrm{~W} / \mathrm{cm}^{2}$ ), we could realize non-invasive and reversible inactivation of the local neuronal activity of the brain in minutes in experimental animals. Furthermore, the laser irradiation increased tissue ATP, adenosine triphosphate, indicating the technique will be useful for protection of neurons from ischemic brain insults. In photo-dynamic tissue oxidation technique using photosensitizing dye, we could reversibly inactivate synaptic transmission of experimental animals by local production of reactive oxygen species, and could control animal behavior. By intensive photo-oxidation of a local area of the cerebral cortex, we could elicit cellular proliferation of novel neural progenitor cells which ubiquitously exist in all over the cortex by propagation of neuronal excitation throughout the cortical hemisphere. Thus, the technique possibly brings out new strategies for regeneration of the central nervous system in patients suffering from various brain in- 
sults. We believe that these photo-dynamic techniques will usher us into a new frontier in treatments for neurological disorders in the central nervous system.

S-1-3

LASER THERAPY FOR PAIN CONTROL

Kazuo Hanaoka M.D.,Ph.D. Kahori Yamamoto M.D. Hideko Arita M.D.,Ph.D. Masaki Nagase M.D.,Ph.D.

Department of Anesthesiology and Pain Relief Center JR Tokyo General Hospital, Tokyo, Japan

Laser therapy, especially, Low reactive Level Laser Therapy LLLT for pain control is very useful and very popular in Japan.Because, this therapy is applied very easily and simply to the pain patient. Besides, in Japan, as the number of the aged is increasing very fast tremendously, we have many chances of treatment of pain patients. It is well known that Stellate Ganglion Block (SGB) is widely used among the aged as sympathetic ganglion block for upper extremities, shoulder, neck, head and facial pain. LLLT therapy, Stellate Ganglion Laser Therapy ( SGL) has same effect of SGB using local anesthetics. LLLT is very safe to the aged compared with SGB in which local anesthetics injection techniques is applied. As you know, the elderly are not able to tolerate the intoxication of local anesthetics sometimes and the potential exists for life-threatening situation to arise more easily with elderly patients. For the SGL, The Superlizer ${ }^{\mathrm{TM}}$ is especially provided not only in Japan but also in Asia, and is very often used in Korea. In this lecture, I would like to talk about the mechanisms of pain relief by laser therapy and applications of LLLT for pain patients.

S-1-4

\section{LOW LEVEL LASER IRRADIATION CHANGES IN INFLAMMATION RELATED SUBSTANCES \\ Masahito Kawatani}

Department of Neurophysiology Akita University, School of Medicine, Akita, Japan

Low level laser irradiation has many actions to cell and/or organ functions. In particular, it became more useful when injury and/or inflammatory condition. These indicate, it could interact with production and movement with inflammation substances or their receptors. Prostaglandins, an inflammatory substance, were synthesized from the epithelium, the smooth muscle, the fibroblast as well as circulated blood cells and immune cells. It dilates the blood vessel, contract the smooth muscle. In addition, excite and inhibit the neuronal activities. Various effects could be apart of many types of prostaglandin receptors, because it have different signal transduction mechanisms. Laser irradiation to the cultured dorsal root ganglion cells did not alter the PGE2 contents in the cultured medium. $\mathrm{KCl}(50 \mathrm{mM})$ activation increased PGE2 contents $(163+24 \%)$. Laser irradiation during the $\mathrm{KCl}$ application to the cells prevented the PGE2 contents increase $(105+18 \%)$. Pre- or post-irradiation was not prevent the PGE2 increase $(172+30 \%, 159+28 \%$, respectably). An NSAID, loxoprofen (0.1-1mM) inhibits the PGE2 contents. It has been reported that PGE2 level was reduced after laser irradiation in rheumatoid arthritis patients. These data suggests that laser irradiation to cells or organs might change the prostaglandins or prostaglandin receptors function.

\section{S-2-1}

\section{LASER PHOTOTHERAPY FOR TREATMENT OF PERIPHERAL NERVE AND SPINAL CORD INJURY (BASIC SCIENCE \& ANIMAL STUDIES)}

\section{Shimon Rochkind}

Director, Division of Peripheral Nerve Reconstruction, Department of Neurosurgery, Tel Aviv Sourasky Medical Center, Tel Aviv University,

Senior Scientist, Neural and Vascular Reconstruction Labs, Ness Ziona, Israel

Laser phototherapy induces nerve cell activation, affects nerve cell metabolism and induces nerve processes sprouting. Our previous studies investigating the effects of low power laser irradiation 632.8 and $780 \mathrm{~nm}$ on injured peripheral nerves of rats have found protective im- 
mediate effects which increase the functional activity of the injured peripheral nerve, maintain functional activity of the injured nerve over time, decrease or prevent scar tissue formation at the injured site, prevent or decrease degeneration in corresponding motor neurons of the spinal cord and significantly increase axonal growth and myelinization. Moreover, direct laser irradiation of the spinal cord improves recovery of the corresponding injured peripheral nerve. A clinical double-blind, placebo-controlled randomized study was performed to measure the effectiveness of laser phototherapy on patients who had been suffering from incomplete peripheral nerve and brachial plexus injuries for 6 months up to several years. This study shows that in long-term peripheral nerve injured patients low power laser irradiation can progressively improve peripheral nerve function, which leads to significant functional recovery. Recently, biodegradable composite transplant based on cell tissue-engineering technology was used for the treatment of complete peripheral nerve and spinal cord injury in rats. The laser phototherapy was applied as a supportive factor for accelerating and enhancing axonal growth and regeneration after reconstructive peripheral nerve and spinal cord procedures. The significance of this innovative methodology will be the provision of new nerve tissue-engineering modality and laser technology for treatment of complete peripheral nerve and spinal cord injury.

\section{S-2-2}

\section{THE ROLE OF PHOTOTHERAPY IN NONHEALING CRURAL ULCERS Junichiro Kubota Kubota Junichiro Clinic, Tokyo, Japan}

Recalcitrant leg ulcers represent a significant problem and present the clinician with special challenges. Chronic leg ulcers have a multifactorial pathogenesis, among which excess and sustained levels of reactive oxygen species (ROS) have been implicated. It is possible that ROS-mediated capillary damage locks the condition of the ulcer in a vicious circle consisting of a detrimental cascade which is self-amplifying. Proteases and pro-inflammatory cytokines exist at elevated levels, and their combined activities keep the wound in the inflammatory phase, with progress into the proliferative and healing stages prevented. The majority of ROS are most likely released by stressed neutrophils and macrophages as part of their attempts to phagocytose and internalize invading organisms and the high level of detritus associated with these wounds. Conditions of localized oxidative stress are thus present in chronic leg ulcers, and the older the ulcer becomes, the more the above factors possibly induce senescence in fibroblasts which react less and less to growth factors. Low incident levels of near infrared (IR) light energy at $830 \mathrm{~nm}$ have been shown to reduce neutrophil-related ROS levels while enhancing the cytocidal and phagocytic activities of both neutrophils and macrophages, and increase the oxygen tension of the irradiated area through an enhanced blood supply. Visible red light at $633 \mathrm{~nm}$ is known to stimulate fibroblast metabolism and enhance blood flow. Combination phototherapy with these two wavebands may provide the key to breaking the vicious circle, controlling inflammation, and enabling chronic ulcers to advance into the proliferative and remodeling phases.

S-2-3

\section{THE IMPORTANCE OF THE MACROPHAGE IN WOUND HEALING Mary Dyson \\ King's College London (KCL), University of London, UK}

During the first day of the inflammatory phase of wound healing, circulating monocytes are attracted to the wound site by soluble mediators and fragments of collagen and fibronectin. Integrins bind the monocytes to the damaged extracellular matrix. Serum factors and fibronectin then mediate their differentiation into macrophages. The macrophages have a two-fold role in wound healing: phagocytosis and secretion. As phagocytic cells they ingest bacteria and fragments of devitalised tissue. The collagenase and elastase they produce assists in the breakdown of matrix degranulation at the wound site. As secretory cells they 
also manufacture and secrete proteolytic enzyme inhibitors and soluble mediators. The proteolytic enzyme inhibitors regulate matrix degradation, limiting its extent. The soluble mediators are involved in the transition from inflammation to proliferation. These mediators consist of a wide variety of growth factors and cytokines including fibroblast growth factor (FGF), platelet derived growth factor (PDGF) and interleukin 6 (IL6). Their activities include recruiting and activating fibroblasts which produce the extracellular matrix of granulation tissue and endothelial cells which produce new capillaries and line larger new vessels during angiogenesis. Lasers and light emitting diodes producing 660,820 and $870 \mathrm{~nm}$ radiation can stimulate macrophages to secrete mediators that collectively stimulate fibroblast proliferation in vitro. If this also occurs when injured tissue is irradiated in vivo, this could be one of the mechanisms responsible for the accelerated transition from inflammation to proliferation when phototherapy is applied to injured tissue using these wavelengths.

\section{S-2-4}

\section{PHOTOTHERAPY FOR POST-RESURFACING WOUND HEALING Mario A Trelles \\ Instituto Médico Vilafortuny/Antoni de Gimbernat Foundation Cambrils, Spain}

Many procedures in the field of -plastic and aesthetic surgery require fairly invasive surgery, and some, like full face resurfacing, leave the patient with a second-degree burn of the entire face. The skill of the surgeon will be for naught, if any of these wounds heals badly due to infection or poor patient compliance. In recent years, low level laser therapy (LLLT) brought speed to wound healing, with excellent results and good patient compliance following the treatments. In more recent years, a new generation of light-emitting diodes (LEDs) has been developed which, although not having the coherence of laser energy, can still offer laser-like photon intensities with quasimonochromatic outputs of a few nanometres on either side of the rated wavelength. This has allowed planar LED arrays to be built into articulated heads, capable of irradiating the full face, or other surgically treated area with awkward contours, in a hands-free manner, thus freeing up the clinician to attend to other patients. The author has been using one such system, the Omnilux series (Photo Therapeutics, Fazeley, UK), which offers three heads of different wavelengths. The blue head at 415 $\mathrm{nm}$ is designed almost exclusively for acne, but the red $(633 \mathrm{~nm})$ and near infrared heads $(830 \mathrm{~nm})$ provide excellent synergy for wound healing, and have even brough full face resurfacing back as a possible treatment due to the rapidly accelerated wound healing and control of other side effects.

\section{S-3-1}

\section{LASERS IN DENTISTRY: A RANDOMISED LLLT CLINICAL STUDY WITH CONTROL GROUP AFTER MULTIPLE TEETH EXTRACTION: PRESENTATION OF SOME SURGICAL CASES WITH HIGH POWER LASER. Zlatko Simunovic ${ }^{1}$, Kresimir Simunovic ${ }^{2}$ \\ 1: Pain Clinic-Laser Center, Switzerland 2: Private Dental Practice, Switzerland}

In recent years there has been increasing number of dentists using laser technology, either low or high-energy level laser. There is no laser yet developed that totally replaces the conventional rotary instruments, but some routine work in dental practice can now be done with use of lasers. Multiple teeth extraction is a dental surgical procedure which, sometimes, followed by complications like haemorrhage, oedema, pain and inflammation, leads to intake of related drugs usually analgesics and antibiotics. The current clinical study was conducted in order to observe the efficacy of phototherapy- LLLT applied during and immediately after multiple teeth extractions. Irradiation Parameters: Total number of patients: 50, randomly allocated to two groups ( $25 \mathrm{p}$-irradiated, energy density applied: $4 \mathrm{~J} / \mathrm{cm}^{2} \mathrm{HeNe}$ laser \& IR Laser 25 p- control group, no laser irradiation). The following parameters were observed: haemorrhage, oedema, redness and pain. The difference was calculated for each pa- 
rameter sorted as follows: absent ( 0 point), mild ( 5 points), moderate (10 points), severe (15 points), the worst (20 points). The difference was calculated and the significance in favour of LLLT $(\mathrm{p}<0.05)$ was demostrated. The difference in number of patients with pain and those who took analgesics was significant between both groups at level of $p<0.001$. Advantages of phototherapy- LLLT are: no bleeding or oedema, no inflammation, significantly reduced intake of analgesic drugs-reduced presence of pain, reduced stay in hospital, fewer incidence of post-oprative complications, no harmful or side effects observed. Effects of phototherapy-LLLT are based upon biostimulative-regenerative effect, anti-inflammatory, analgesic anti-bacterial and anti-viral effects.

S-3-2

\section{POST SURGICAL PAIN CONTROL WITH LLLT Mario Trelles \\ Instituto Médico Vilafortuny/Antoni de Gimbernat Foundation Cambrils, Spain}

Pain is a major factor after any moderate to major surgical procedure, and control of postoperative pain is a considerable problem, to avoid the patient's becoming over-dependent on narcotic analgesics. LLLT, or laser therapy, has an excellent history in pain control of all pain types, acute and chronic, with $830 \mathrm{~nm}$ appearing to be the wavelength of choice. A new light-emitting diode (LED)-based system offers a large area articulated $830 \mathrm{~nm}$ head, which typically delivers $60 \mathrm{~J} / \mathrm{cm}^{2}$ over a 20 minute treatment session. The author has experimented with this head following a variety of surgical procedures, and the results have been very interesting. Full face resurfacing patients, in a comparative trial comparing LED-treated with untreated patients, pain was reduced by more than $75 \%$ or average, and the post-treatment analgesic requirements were cut by more than one-half.. The author believes that LED phototherapy for pain will become an indispensable part of the plastic surgeon's armamentarium, in addition to pain clinics of all kinds.

S-3-3

\section{PAIN CONTROL IN PHYSIOTHERAPY AND SPORTS MEDICINE G David Baxter \\ School of Physiotherapy, University of Otago, New Zealand}

A range of modalities may be employed for the non-pharmacological management of musculoskeletal pain, ranging from ancient techniques such as acupuncture, to more modern electrophysical agents, such as transcutaneous electrical nerve stimulation (TENS), pulsed electromagnetic energy (PEME), and low intensity laser therapy (LILT). Physiotherapists and sports medicine specialists currently employ a variety of these pain-relieving modalities alongside other treatments such as manual and exercise therapy as part of a comprehensive approach to patient rehabilitation. Laser therapy or LILT can potentially provide a safe and effective means of pain control for physiotherapy and sports medicine, depending upon application of appropriate irradiation parameters based upon an evidence-based approach to parameter selection. Current evidence suggests that best results for pain control are obtained with the use of infrared wavelengths $(800-950 \mathrm{~nm})$, treatment dosages of at least 1-3 J per point, and a systematic approach to treatment (comprehensive treatment of sites, early and regular treatments). In contrast, poor results have typically been reported to be associated with lower wavelengths and irradiance levels, and inappropriate treatment methods (e.g. non-contact treatment). While the mechanisms underlying the observed pain relieving effects of laser therapy in musculoskeletal pain are still a matter of some debate, it is clear that such mechanisms include localised and peripheral effects, as well as more systemic effects throughout the central nervous system. As with any other form of treatment, optimal results are obtained with selection of appropriate treatment parameters, and with due recognition of the scientific basis of this modality. 


\title{
S-4-1
}

\author{
ADJUVANT STRATEGIES OF PDT \\ Ahn, Woong Shick, M.D., Ph.D. \\ Department of Obstetrics \& Gynecology., KangNam St. Mary’s Hospital, \\ The Catholic University of Korea, Seoul, Korea.
}

Photodynamic therapy (PDT) has been reported to be effective for treating various tumors and to induce apoptosis in many tumor cells. Numerous preclinical studies have demonstrated enhances the host immune response, however, the mechanisms behind this enhancement are little known. Results from preclinical and clinical studies conducted worldwide over a 25-year period have established photodynamic therapy as a useful treatment approach for some cancers. Even though PDT has powerful effects for the treatment of tumors in vitro and in vivo, they need to improve their efficiencies using adjuvant modality including gene therapy, cyclooxygenase-2 inhibitors treatment, matrix metalloproteinase inhibitors, angiogenic inhibitors, anti cancer agent etc. Our laboratory also doing combined modality approach using adenovirus mediated $\mathrm{p} 53$, interleukin-12, immunomodulator (ODN), antioxidant (EGCG, catechin) and arsenic compound $\left(\mathrm{As}_{2} \mathrm{O}_{3}, \mathrm{As}_{4} \mathrm{O}_{6}\right)$ etc. All the experiment results showed PDT plus AdCMVp53 gene therapy had more potent antitumor effects on human cervical cancer cells, with induction of apoptosis at least through activation in $\mathrm{p} 53$ protein at the cellular and tumor tissue levels. Tumors growth significance inhibited in mice treated with AdmIL-12+PDT. PDT-generated cell lysates + ODN codelivery could be an effective approach to induce CTL immune responses as a possible immunotherapeutic strategy for cervical cancer. Antioxidant also showed much synergistic effects with PDT. Conclusively adjuvant strategies with photodynamic therapy enhances photodynamic therapy treatment efficacy in tumors.

S-4-2

\section{PHOTODYNAMIC THERAPY FOR LUNG MALIGNANCY}

Tetsuya Okunaka*, Jitsuo Usuda and Harubumi Kato

*Pulmonary Disease Center, Sanno Hospital, International University of Health and Welfare, Department of Surgery, Tokyo Medical University, TOKYO, Japan

Photodynamic therapy (PDT) has now achieved the status of a standard treatment modality for centrally located, early-stage lung cancer. Over the past decade, 263 lesions with central-type early-stage lung cancers have been treated with PDT in Tokyo medical University hospital. Overall complete remission was obtained in $84.8 \%$ of the total number of lesions. Adjuvant photodynamic therapy before surgery for advanced bronchogenic carcinoma was also expected. Thirty-two lung cancer patients were received the preoperative PDT for the purpose of either reducing the extent of resection or increasing operability. The initial purpose of PDT, i.e. either to reduce the extent of resection or convert inoperable disease to operable status, was achieved in 27 out of 32 patients treated. Although quite recent, treatment-using PDT has been introduced for the first time in-patients with peripheral lung cancer, who did not previously meet the criteria for surgery. Ten patients underwent this trial, of which 7 achieved partial remission. No serious complications except for 2 case of pneumothorax, were noted. As increasing number of patients consider quality of life after therapy, the indications for PDT are expect to expand. The success in clinical trials of PDT for cancer treatment offers encouragement for its future use. 


\section{S-4-3}

\section{THE FEASIBILITY OF PHOTODYNAMIC THERAPY FOR HEMANGIOMA WITH TALAPORFIN: A CHICKEN COMB MODEL}

Takafumi Ohshiro ${ }^{1}$ Toshio Ohshiro ${ }^{2}$ Tatsuo Nakajima ${ }^{3}$ Katsumi Sasaki $^{1}$ and Syunji Fujiii ${ }^{1}$

1: Ohshiro Clinic, Tokyo, Japan; 2: Japan Medical Laser Laboratory, Tokyo, Japan; and 3: Keio University, Tokyo, Japan

. Talaporfin sodium (mono-L-aspartyl chlorin e6) is a novel photosensitizer, and is currently being used for photodynamic therapy(PDT) for early lung cancer in the combination of irradiation of the $664 \mathrm{~nm}$ laser. The characteristic of Talaporfin sodium is that the skin photosensitivity after injection of this drug disappears faster than existing photosensitizers. This study is concerned primarily with the vascular events that occurs post irradiation in the chicken comb as a hemangioma model after sensitization with Talaporfin sodium. The animals received single intervenous bolus injections of Talaporfin sodium, and a $1 \mathrm{~cm}$ diameter area of the comb of each animal was irradiated with $664 \mathrm{~nm}$ laser. The gross changes in chicken combs were recorded for 7-14 days after PDT. For histologial examination, H.E., PTAH, AZAN stained sections were analyzed. All treated chicken combs were blanched after PDT. Microscopy demonstrated an absence of erythrocyte and the vessel lumens were obliterated, while leaving the normal overlying epidermis completely intact. .In the chicken comb model, PDT with Talaporfin sodium may be a feasible method to treat dermal hypervascular lesions.

S-4-4

PDT FOR GASTROINTESTINAL TUMORS

Tetsuya Nakamura ${ }^{1}$, Hidetsugu Yamagishi ${ }^{2}$, Takeshi Oinuma ${ }^{2}$, Hideyuki Hiraishi ${ }^{2}$ and Akira Terano ${ }^{2}$

1: Department of Endoscopy; and 2: Department of Gastroenterology, Dokkyo Medical University School of Medicine, Tochigi, Japan

PDT is a safe and effective treatment; however its indication for gastrointestinal (GI) tumor is limited to superficial early esophageal and gastric cancer in Japan. Endoscopic mucosal resection (EMR) is the first choice of the treatment for intra-mucosal GI tumors. Therefore, we have designed a new therapy called "modified PDT" to treat GI tumors which is relatively invasive and not indicated for EMR. Methods: The major point of modified PDT is as follows. Irradiation of pulsed excimer-dye laser $(4 \mathrm{mj}, 60-80 \mathrm{~Hz}$, Hamamatsu Photonics Inc., Japan) is applied to tumors 48 and 72 hours after Photofrin? $(2 \mathrm{mg} / \mathrm{kg})$ injection. Between November 2002 and September 2005, this procedure was carried out in 20 patients (mean age 73 years, range 55 to 87 ) who were not indicated for EMR in our hospital. Written informed consent was obtained from all patients. Results: Nine of ten early gastric cancers, four of six early esophageal cancers and one rectal cancer were completely disappeared during 6 to 40 months follow-up period. Three advanced gastric cancers were partially response. No serious side effect was occurred. The modified PDT is remarkably effective and useful in the treatment of GI tumors not indicated for EMR. This technique may be considered an alternative therapy for not only esophageal and gastric cancer but also rectal cancer.

\section{S-4-5}

Necrosis depth in interstitial photodynamic therapy varied nonlinearly as function of light dose and photosensitizer dose for a solid tumor model

Woong-Sik Ahn, ${ }^{1}$ Soo-Mee Bae, ${ }^{1}$ Ki-Hong Kim, ${ }^{2}$ Jong-Ki Kim ${ }^{2 *}$

${ }^{1}$ Department of Gynecology, Catholic Medical Center, Seoul, Korea and '2Departments of Radiology and Biomedical Engineering, Catholic University Hospital of Daegu, Korea

In order to prepare a protocol for interstitial photodynamic therapy, the relation of necrosis depth of a solid tumor with light/sensitizer dosimetry was investigated. A solid tumor was induced by injecting TC-1 tumor cell line subcutaneously into C57BL1/6 mouse. Photogem (Hematoporphyrin derivative, Russia) was injected via tail vein with dose of $1-5 \mathrm{mg} / \mathrm{BWKg}$. 
When tumor was grown as much as $1 \mathrm{~cm}$ of diameter, an interstitial light diffuser was implanted in the center of tumor, and irradiated with different light dose using a $630 \mathrm{~nm}$ Diode Laser: 50-200 J/cm2. Injected potosensitizer was extracted from tumor tissue without irradiation and their tissue-accumulated quantities were measured with a fluorescence spectrometer. Necrosis and apoptosis areas were measured from the PDT treated tissue at fourth or 8th days after irradiation using hematoxylin and eosin staining and tunnel assay, respectively. Although the maximum uptake of photosensitizer occurred at $4 \mathrm{mg} / \mathrm{BWKg}$, the necrosis area was larges at $3 \mathrm{mg} / \mathrm{BW}$ with irradiation of $100 \mathrm{~J} / \mathrm{cm} 2$. However maximum necrosis was observed at $150 \mathrm{~J} / \mathrm{cm} 2$ when Photogem was injected at $3 \mathrm{mg} / \mathrm{BWKg}$. These findings suggest actual efficacy of interstitial PDT is complex function of light and drug dose. Photobleaching and excessive absorption of light by more concentrated tissue- photosensitizer may be a source of these apparent nonlinear behaviors. Therefore it is necessary to prepare clinical protocol for interstitial PDT with appropriate model study.

\section{S-5-1}

\section{LLLT AND PHOTOTHERAPY IN ITALY}

\section{Leonardo Longo, MD}

\section{Institute for Laser Medicine, Siena University - Florence}

The first treatment of LLLT in Italy was made in 1975, in Florence, for the healing of a patient with decubitus ulcer. For the 31 years since then, we have continued to use non surgical lasers for many diseases. Different types of laser visible and near infrared were used, alone or in association with other radiations, as magnetic field and electricity. Different procedures were followed. Some experiences were published on this topic, following the famous rules of the clinical research, as Helsinki Declaration and other similar protocols. Today the anti-inflammatory effects of LLLT on the tissue irradiated are generally accepted, as the effects on wound healing. Many people in Italy use lasers for the treatments of rheumatologic diseases and for sport traumas of bones, muscles, tendons and joints. The analgesic effect of lasers on neuralgias has also been reported by many Authors. In aesthetic medicine, LLLT and intense pulsed light systems are used frequently with the procedure of the non ablative rejuvenation, for wrinkles, cutaneous dystrophies, hypertrophic scars, stretch marks. But lasers and light could have effects also on the body metabolisms and on the energy circulation of the body. Evidence based medicine already showed positive effects of laser beams about the treatment of diabetic patients, and on paraplegic patients. Aim of our study is to select the diseases where some laser beam have effect and to investigate further diseases where the effects of the lasers and light could be useful. We started the diabetic patients' treatment with an experimentation as phase 2 of the clinical experimentation's schema. 10 patients were enrolled in this experience, 5 with diabetes type 1 and 5 type 2 . We used the same diode laser system coupled with magnetic fields but using different procedures of treatment. The glycaemia was measured immediately before and 10 minutes after each session of treatment. Glycate haemoglobin levels were measured before and three months after the treatment. In another experience we treated some paraplegic patients, after medullar traumatic lesions, knowing that laser beams have regenerative effect on neurons in vitro. Some months ago we also started an experimentation with endovenous LLLT treatment, called histodialysis. We put an optical fibre in a needle then we put the needle in a vein. We irradiated until all the blood of the patient flew in that vein. This procedure was proposed by Russian Authors many years ago, for the treatment of immunological diseases and disorders. We treated patients with AIDS, hepatitis and rheumatoid arthritis. The results obtained were largely positive. The follow up was variable depending of the type of the disease and the behaviour of the patient after the treatment. Larger number of patients must be treated and followed for some years, for to obtain definitive data. In Italy lasers and pulsed light are largely used by Physical therapists, dermatologists, geriatricians and neurologists. Our data demonstrate that the usual diabetes treatment could be totally changed in the near future, especially if the results of laser treatment will be also positive during next phases of the exper- 
imentation. The action mechanism of this treatment must be studied. The positive effects on paraplegic patients and neurological system open another field of application of these instrumentations. The histodialysis is a procedure that could give further positive results on many diseases now less treatable with other treatment.

\section{S-5-2}

THE CURRENT STATUS OF LASER TREATMENT OF DERMATOLOGY IN VIET NAM Tran Hau Khang, and Nguyen Si Hoa

National Institute of Dermato-Venereology Ha noi, Viet nam

LASER technology has become an invaluable tool to advance therapies. Lasers in dermatology have become trusted allies in resolving major problems in a wide range of indications. In Vietnam, LASERs have been recently used to treat skin diseases in almost dermatological units at provincial level. The CO2 LASER and He-Ne LASER are available in Dermatological hospitals/dispensaries/Centers. They are used to treat mainly skin diseases such as warts, acne, keratosis and condyloma acuminata. At the national level, the Institute of Dermato-Venereology (NIDV) is equipped with the most advanced laser technology including Carbon dioxide (CO2) laser, the YAG (Yttvium aluminum garnet) laser, KTP.... By proper selection of laser parameters such as pulse time, intensity and frequency at suitable wavelengths, we have managed the following skin diseases:

1. Laser resurfacing: Wrinkles, scars, blemishes of various types.

2. LASER tattoo removal: This is not one time treatment. Depending on the type of tattoos three to six treatments was required.

3. Laser skin peeling: Reduced wrinkles around the lips, the eyes, softening fine of winkles.

4. Laser removal of birthmarks: Port-wine stain birthmarks respond well to this therapy. The abnormal blood vessel is reduced in size.

5. Other laser treatments:

- Sebaceous hyperplasia.

- Various types of benign growth on the skin surface.

- Facial scar removal: Scars occur as a result of surgery or trauma were not completely eliminated, but they were greatly modified with this treatment.

- Sun damage, brown sport: Scaly red sun spots and brown blemishes were also removed.

- Warts, condyloma acuminate removal.

Laser has revolutionized the practice of medicine. The use of laser technology in the treatment of skin diseases is not new, but it is being constantly expanded and refined. We are using the bases of laser and developing the future in this area.

\section{LOW LEVEL LASER THERAPY AND LIGHT THERAPY IN KOREA Jin Wang Kim, ${ }^{1}$ Joung Ok Lee ${ }^{2}$, 1; Hallym University College of Medicine, Department of Plastic Surgery; and 2; Department of Dermatology}

All of the skin rejuvenation, acne treatment, scar removal with laser or quasilaser noncoherent light source closely related with skin wound healing, how ever the wound healing period is nor exactly confirmed but the theory. Recent trend laser therapy of light therapy is target cell such as mast cell (low level laser assisted liposuction), macrophage \& peri-endocyte (wound healing with $830 \mathrm{~nm}, 633 \mathrm{nmLED}$, Bioptron etc), micochondria originated ATP production enhaned collagen production ( IPL, 633nm LED,LLLT) and PDT assisted acne and acne acar removal ( $415 \mathrm{~nm}$ LED, $633 \mathrm{~nm}$ with ALA) and aquaphoresis.. For the aesthetic purposes we used $415 \mathrm{~nm}$ LED , and $830 \mathrm{nmLED}, 633 \mathrm{~nm}$ LED as adjunctive therapy..Specific wavelength and specific energy produce different action. For the aesthetic purposes we used $433 \mathrm{~nm}$ LED , and 830nmLED, $633 \mathrm{~nm}$ LED as adjunctive therapy. For 100 patient with skin disease including photoaging, after and during wound management for aesthetic purpose. We used the LED light 60 joule/ $\mathrm{Cm}^{2} \mathrm{LLLT} 5$ joule/ $\mathrm{Cm}^{2}$ and polarized light therapy (400-2000 nm, $40 \mathrm{~mW} \mathrm{~cm}-2,2.4 \mathrm{~J} \mathrm{~cm}^{-2}$ ) until complete closure. In 
wound healing, acne removal, scar treatment, pain and photorejuvenation. The result is very positive and it's main action is as follows

1. oxygen supply increase (photobioactivation)

2. After $\mathrm{O}_{2}$ supply, production of SOD destroys rapid growing tumor cell destruction.

3.Benificiary to keloid and hypertrophic scar and good for skin rejuvnation,acne

PDT lamp with or without photosensitizer treats the acne bacterium Propionibacterium acnes (P. Acnes).

Very effective treat MRSA,,pyogenic granuloma gentian -Violet with $633 \mathrm{~nm}, 415 \mathrm{~nm}$

Stimulating preexisted fibroblast in photoaging with $633 \mathrm{~nm}$ is very effective, but it is more important in laser skin resurfacing effect with combination use of ablative with a nonablative light source. Pain and anti-inflammatory photobioactivation cascade both appear in the same period so it is beneficiary to treat patient with less painful procedure The satisfaction rate is very high in korean patients with various light source therapy and the low level laser and light energy therapy is very Important and accepted method of treatment tool now in pain control, wound healing, inflammation control. LLLT and LED therapy is bioenergy ubiquitous.

S-5-4

\section{LLLT AND PHOTOTHERAPY IN THAILAND} Narong Nimsakul, M.D.

Director, Institute of Modern Medicine, Bangkok Thailand. Honorary President, International Society for Laser Surgery and Medicine

Thailand started to introduce Laser Technology into Medical fields in 1979, the first laser was $\mathrm{CO}_{2}$ Laser of 50 Watts output (Sharplan7 91), the author was the first surgeon to use $\mathrm{CO}_{2}$ laser in plastic \& reconstructive surgery, by transferring technology from Japan. The Thailand Society for Laser Medicine \& Surgery was founded in 1988, became active member of the International Society for Laser Surgery and Medicine, and of the Asian-Pacific Association for Laser in Medicine and Surgery. Thailand hosted the 10th Congress, International Society for Laser Surgery and Medicine in November 12-17,1993; and hosted the 9th Congress, Asian-Pacific Association for Laser Medicine and Surgery in October 18-20,2002. Thai Physicians and surgeons have been trained in EU, USA, and Japan, and most of them still using high power systems for surgery and non-invasive procedures in various field. Very limited uses of LLLT and phototherapy are the present situation. Only one company manufacturing the $\mathrm{CO}_{2}$ Laser of 25 watt Output. The author will demonstrate the applications of He-Ne Laser, Diode Laser and low-power $\mathrm{CO}_{2}$ Laser in the field of plastic and reconstructive surgery.

\section{S-6-1}

\section{YEARS CLINICAL EXPERIENCE IN LASER TREATMENT OF TINNITUS} Miroslav Prochazka, M.D. Private Rehab Clinic, Prague, Czech rep.

In time from 1993-2006 we work with non invasive laser therapy of tinitus, published more articles about treatment of this widespread civilisation disease. In long-term results we can say, that after laser treatment 26 per cent of patients are totally free of tinnitus. 43 per cent of patients have relief of tinnitus more as 50 percent (monitored on three original scales for best results from the point of the quality of life, very importnant for patients with this disease). Only 16 per cent of patients have no effect. Our results enable us to pinpoint non invasive laser - with necessary parameters of laser probe used. It means sufficient power output $/ 300 \mathrm{~mW}$ ), wavelenght (infrared, $830 \mathrm{~nm}$ ) and the direction and localisation of laser beam.After more than 13 years of use non invaseive laser therapy of tinitus we have seen no side effect of this therapy. 


\section{THE BENEFITS OF PHOTOTHERAPY-LLLT IN THE ORAL CAVITY AND MAXILLOFACIAL DISEASES \\ Zlatko Simunovic, MD, FMH, Pain Clinic-Laser Center, Switzerland Kresimir Simunovic, DMD, Private Dental Practice, Switzerland}

In the initial part of our research we have conducted and randomized controlled animal study, where we have evaluated the effects of laser irradiation on the wound healing of surgical wounds on rabbits. Wound healing by a full skin thickness incision on $\mathrm{n}=68$ rabbits randomly divided in four equal groups $(n=17)$ according to the type of laser irradiation (1. Control 2. HeNe 3.IR 4. $\mathrm{HeNe}+\mathrm{IR}$ ) Photographs of the wounds were taken the different days and wound areas were analysed by ANOVA computer and tissue samples trough the wound site were taken for histology and tensile strength determination. we observed that $\mathrm{HeNe}+$ I.R. $904 \mathrm{~nm}$ Laser irradiation accelerated the speed of the scaring with significant effects on tissue tensile strength, epidermal growth, rate of wound contracture, collagen formation and lymphocyte concentration, rushing regression of post-operatory oedema with pain relief, which proves photobiostimulating effects. The ways of application and the effects of LLLT on human body are analogical to those of similar physiological structure in animal tissue. In the case when you radiate mucous membrane of oral cavity i.e. subtle tissue without corneous stratum, the mucous area is able to absorb better and quicker the laser rays, and for this reason the total energy rate of rays is significant lower and still with positive effects. We report $a$ new treatment possibility of oral cavity diseases like of gingivitis, periodontitis, stomatitis, wounds after extractions, recovery after dental and periodontal surgical procedures, open and closed wound healing after minor and major oral surgery, lip wounds and combustions, haemathoma, oedema, scar tissue, herpes labialis, aphthae, rhagades, etc. This lecture will give you a brief overview of dental disorders where phototherapy proves to be an efficient therapeutic procedure with exact irradiation technique and efficient energy densities (dosimetry) in the clinical applications of phototherapy-LLLT in dentistry. Thanks to the lasers well known biological effects and to the restauration of the oral biological equilibrium we noted in the case of the oral cavity diseases mentioned above relatively rapid regression of the inflammation with the corresponding lessening of secretion, swelling and haemorrhage and specific antalgic effects. Also we noted an anti-bacteriological and anti-viral effect and tissue regeneration.

S-6-3

\section{PHOTOTHERAPY AND IMMEDIATE EFFECTS IN GINGIVITIS AND MARGINAL PERIODONTITIS}

\section{Dr. Rozalia Dana Vieru1, Dr. Stana Paunita ${ }^{2}$, Dr. Anca Dumitriu ${ }^{3}$, Prof. Dr. Horia Traian Dumitriu ${ }^{4}$ \\ 1: Periodonthology Cathedra "Carol Davila" Medical and Pharmacy University, 2: Periodonthology \\ Cathedra "Carol Davila" Medical and Pharmacy University, Bucharest, Romania; 3: Periodonthology \\ Cathedra "Carol Davila" Medical and Pharmacy University, Bucharest, Romania; and 4:Periodonthology Cathedra "Carol Davila" Medical and Pharmacy University, Bucharest, Romania.}

Biostimulation therapy by LLLT associated with classic treatment increase the percentage of success and shortens the healing time in gingivitis and periodontitis. To evaluate the effectiveness of laser therapy LLLT in gingivitis and marginal periodontitis. 37 patients with gingivitis and marginal periodontitis were asked to participate to the research. 13 patients were treated classically (control group) and 24 (the experimental group) benefit of laser. A Diode Laser was used, from [3] BTL 10 (Czech Republic) with 830 nm, class 2B, class 1, tip BF (EN60601-1). We followed several parameters: the time for hemostasis and formation of the cloth, the pain relief time, percentage for recurrences and the use of other medication or not. We compared the classical treatment with the laser therapy by following few parameters of the immediate effects. We found from our clinical observation that the immediate effects reduced the bleeding up to $80 \%$, inflammation and pain relief up to $90 \%$ and for the long term effect we found no post-treatment alteration. The recurrence was a much smaller $20 \%$. Use of chemical medication is decrease. We got a more sure 
and safe compact bone mineralization. Laser Therapy brings improvement to the treatments, reduce the time of healing, the use of medication and the pain, recurrence appear in a lower percentage

S-6-4

THE EVOLUTION OF PHOTOTHERAPY IN THE ORAL MEDICINE FIELD Luciana Almeida-Lopes

ElectroThermoPhotoTherapy Laboratory, UFSCar, SC, Brazil

Nowadays the technology developed for Photo Therapy equipments have been evolving rapidly, however the work techniques and the treatment protocols have not been taken the adequate advantage of this new order of things. The aim of this study is to point out the current status of the Photo Therapy in the Oral Medicine Field, talking about the use of lasers and LEDs systems and discussing about the adjustable parameters such as: laser emission, deposited energy used, fluency, laser out put power, irradiance, and the way it can interfere on the clinical results. We are going to present also polemic themes which should be discussed to exhaustion in order to definite intrinsic variables of a treatment protocol such as the evaluating of the fluency calculation and the establishment of the correlation between ideal fluencies obtained from in vitro models and the ones to be used in vivo. We will talk about the definition of the treatment posology that comprehends total energy deposited, maxim fluency admitted, number of applications points, frequency and sections number, higher fluency levels and irradiance used nowadays, what implies in deeper penetration and different kinds of interaction.

S-7-1

Nd:YAG LASER APPLICATION IN TRACHEO BRONCHIAL TUMORS B. Krishna Rau ${ }^{1}$ and S. Krishna ${ }^{2}$

1: Professor Emeritus, Dr, MGR Medical University and 2:Consultant Surgeon, Devaki Hospitals, Chennai, India.

Ninety three patients with obstructing tracheobronchial tumors were treated with Neodymium Yttrium - Aluminum - Garnet (NdYag) laser photocoagulation over a period of six years. There were 67 Males and 26 Females with a mean age of 44.3 years (range 6- 79 years). 21 benign and 72 malignant lesions were treated with a total 212 sessions of laser photoabalation (mean 2.4 sessions). The anatomical distribution of lesions was as follows; larynx 9 ( 3 benign and 6 malignant) trachea 39 ( 27 benign and 12 malignant) left main bronchus 27 (14 malignant) right main bronchus 24 (14 malignant) and vocal cords -9 ( 3 malignant) There were 21 patients with squamous cell carcinoma, 2 adenocarcinomas, 1 adenoid cystic carcinoma, 7 cases of locally infiltrating tumors, 6 cases of carcinoid tumor and 16 benign lesions. 21 patients had the tracheotomy tube in place when treatment was started. 18 of the 21 patients with tracheotomies were weaned off the tube in a mean of 5.5 days from the start of the treatment. Tracheal lumen was restored in 31 (79.4\%) patients. In the other 8 (20.6\%), lumen was achieved, but not sustained. Complications included bleeding in three cases which were managed conservatively, two cases of pneumothorax, and four cases of bronchospasm. There were six deaths during the follow up but none attributable to the procedure. Laser photocoagulation offered effective treatment in patients with obstructing tracheobronchial tumors, with acceptable morbidity. For the past six years, self expanding metal stents [SEMS] are positioned in all malignant tracheo bronchial tumors.

\section{S-7-2}

\section{ISCHEMIC HEART DISEASES TREATMENT BY MEANS OF INTRAVENOUS HeNe LASER BLOOD IRRADIATION COMBINED WITH PHYTOTHERAPY}

Hayk S. Arakelyan 1,2, Ruzan A. Durgaryan ${ }^{1}$

Panarmenian Association of Laser Therapy and Integrative Medicine, University of Traditional Medicine

To compare therapeutic efficiency of Combined Intravenous HeNe Laser Blood Irradiation (IV

HeNe LBI) and Phytotherapy (PhT) with separate IV HeNe LBI and PhT for the treatment of the 
patients with Ischemic Heart Disease (IHD). For this purpose, 39 man patients have been studied with diagnosis of stabile, 3 rd functional class angina pectoris established by Tred-mill Electreocardiography. They have been divided into the following 3 groups: 1.13 patients have been treated with combination of IV HeNe LBI and PhT - Hawthorn and Peppermint ( Group 1); 2.13 patients have been treated with IV HeNe LBI (Group 2) 3. 13 patients have been treated with PhT - Hawthorn and Peppermint (Group 3). There was no significant difference in mean ages, blood pressure (BP), serum low-density lipoproteins (LDL), high-density lipoproteins (HDL), coagulographic parameters among 3 groups but there were typical pathological changes for IHD. IV HeNe $(630 \mathrm{~nm}) \mathrm{LBI}$ has been done outputing power at the end of light-guide inserted into a vein from 1 up to $3 \mathrm{~mW}$, exposition beginning from 20 reaching till 60 minutes. Procedures were conducted on a daily base, 10 sessions on a course of therapy (3 courses in general). For PhT has been used Hawthorn and Peppermint. After treatment course BP, biochemical blood parameters - LDL, HDL, coagulographic parameters and functional class of angina pectoris (by Tred-mill Electrocardiography) have been restudied and the highest improvement of the mentioned parameters has been noticed in Group 1 then Group 2, and Group 3.

\section{S-7-3}

RAPID IMPROVEMENT OF SYSTEMIC AND LOCAL MICROCIRCULATION AFTER IRRADIATION OF DIABETIC PATIENTS WITH POLYCHROMATIC VISIBLE AND INFRARED (IR) LIGHT

\section{K.A.Samoilova ${ }^{1}$, M.A.Menshoutina ${ }^{2}$, E.Yu.Vasina ${ }^{2}$, N.A.Zhevago ${ }^{1}$, V.V.Achkasova ${ }^{2}$,} N.N.Petrishchev ${ }^{2}$, A.G.Ryabinin ${ }^{1}$

1. Institute of Cytology, Russian Academy of Sciences, and 2. I.P. Pavlov Federal Medical University, St.Petersburg, Russia

Up to now it has remained unclear whether the solar polychromatic visible (VIS) and near IR radiation has the therapeutic activity that is characteristic of laser light. A possibility of improvement of microcirculation in skin microvessels of the dorsal surface of the higher and lower extremities of 50 patients with type 2 diabetes mellitus complicated with polyneuropathy has been studied by the high freaquency ultrasound dopplerography. After two daily irradiations with VIS or VIS + IR polarized light (385-750 nm or 385-1700 nm, $95 \%$ polarization; power and energy density of $40 \mathrm{~mW} / \mathrm{cm}^{2}, 12 \mathrm{~J} / \mathrm{cm}^{2}$, phototherapeutic devices Q-light, Switzerland). At $2 \mathrm{~min}$ after irradiation of the sacral area the volume blood flow rate (BFR) in hand skin microvessels increased by $20-29 \%$, reached maximum $(+32-35 \%)$ in $30 \mathrm{~min}$, decreased after $24 \mathrm{~h}$, and increased again after the $2^{\text {nd }}$ irradiation. This indicated an improvement of microcirculation at the systemic level. Irradiation of feet was accompanied by an equally rapid increase of local BFR with maximum $(+41-47 \%)$ in $30 \mathrm{~min}$. The mean increment of BFR at the regional and systemic levels was similar, and so were effects of the VIS and VIS + IR lights. The increase of BFR developed on the background of a decrease of its sensitivity to acetylcholine (ACh) and nitroglycerin (NG) solutions administered intracutaneously by ionophoresis. This means that the light affects both endothelium-dependent and endothelium-independent vasodilatation. At $2 \mathrm{~min}$ after the direct and distant light action the degree of the BFR increase exceeded that 2 min after an intracutaneous administration of $0.1 \% \mathrm{NG}$ and was compatible with the maximal increment value after ionophoresis of $0.3 \%$ Ach. It is suggested that the light initiates the appearance in circulation of endogenous vasodilators, first of all, of nitric oxide.

S-7-4

PHOTOTHERAPY, A MANDATE IN SURGICALLY TREATED VITILIGO

Sherif S. Awad, MD, PhD, Moetaz M. El-Domyati, MD, Tag El-Din Anbar, MD

Department of Dermatology \& Venereology, Faculty of Medicine, El-Minya University, Egypt Phototherapy techniques were introduced by the Egyptians in the old and modern history of medicine for the sake of managing several maladies. Phototherapy is considered a corner stone in the management of arduous vitiligo, yet failures are well known. Recently, the introduction of surgical techniques provided a new frontier in the management of vitiligo and 
replacing other conventional unsuccessful therapies. The aim of this work is to clarify whether phototherapy which failed to resolve the vitiligo problem in patients is still required in the strategy of surgical management of the same cases or not. Thirty vitiligo patients, non-responding to conventional phototherapy, were surgically treated. Three main surgical procedures were introduced for management; Ultra-thin Theirsch grafts, Suction blister grafts and mini-punch grafts. Phototherapy using UVA or UVB (Broad band and Narrow band) was used post-operatively whenever required. Phototherapy acted as a stimulator for melanocytic proliferation and function and/or as an immune-suppressant halting the melanocytic destructive process. Thus, helping in completion of success of treatment and proved to be essential for obtaining satisfactory results. Different doses and number of sessions were applied according to the type of the surgical procedure and the phototherapy regimen used in each case. In conclusion, phototherapy is a mandate in the treatment of surgically treated vitiligo, without it unsatisfactory results can again be the outcome.

S-8-1

\section{THE ROLE OF PHOTOTHERAPY IN SPORTS INJURY G David Baxter \\ School of Physiotherapy, University of Otago, New Zealand}

Phototherapies, including low intensity laser therapy (LILT), monochromatic light therapy, and visible polarised light therapy, can potentially represent safe and effective treatments for the management of various types of musculoskeletal sports injuries. The principal effects of these modalities include the photobiostimulation of tissue repair mechanisms, and the relief of musculoskeletal pain. Acceleration of healing in parallel with pain control makes phototherapies (and laser therapy in particular) an attractive option for use in sports rehabilitation, especially in comparison to other electrophysical agents which typically produce a single effect (e.g. ultrasound for tissue repair, electrostimulation for pain relief). Furthermore, the athermic nature of phototherapies mean that these treatments can be employed in the earliest, most acute stage of injury, without the potentially adverse effects typically associated with those treatments which may lead to heating (e.g. ultrasound). However, phototherapies need to be used as part of a comprehensive approach to the rehabilitation of the injured athlete, and their effectiveness in the treatment for sports injuries depends upon application of appropriate irradiation parameters, based upon an evidence-based approach to parameter selection, and due consideration of the biophysical principles underpinning such treatments.

\section{S-8-2}

\section{PHOTOTHERAPY IN THE TREATMENT OF SPORTS-RELATED TEMPOROMANDIBULAR JOINT INJURY Junichiro Kubota \\ Kubota Junichiro Clinic, Tokyo, Japan}

As participation in sports activities increase, so do sports-related injuries. Trauma to the temporomandibular joint (TMJ) can cause the affected person a great deal of pain as well as negatively influencing the patient's quality of life (QOL). Low incident levels of diode laser irradiation have been very effective in relieving TMJ joint pain. The present study reports on 16 representative cases of TMJ pain treated with a GaAlAs diode laser, $830 \mathrm{~nm}$ continuous wave, $1000 \mathrm{~mW}$, irradiance $669 \mathrm{~mW} / \mathrm{cm}^{2}$, for 10 to $15 \mathrm{sec} /$ point in the defocused noncontact mode (radiant flux 6.7 or $10 \mathrm{~J} / \mathrm{cm}^{2}$ ), once per week. Twelve of the patients had chronic and 4 had acute pain, with unilateral pain in 14 and bilateral pain in 2 subjects. Acute pain required an average of 2 sessions, whereas chronic cases required an average of 11 sessions. TMJ pain and related TMJ disorders such as trismus were successfully treated in 15 patients, with incomplete resolution in only one of the chronic pain subjects. The recent development of a new generation of powerful quasimonochromatic LED-based $830 \mathrm{~nm}$ systems has offered an interesting hands-free tool to irradiate large areas of tissue in one session, which may well 
offer the sports practitioner an even more convenient, safe and effective method for pain relief in patients with sports-related TMJ pain, or other pain entities.

\title{
S-8-3
}

\section{EFFECTS OF DIODE LASER IRRADIATION ON SUPERFICIAL BLOOD FLOW IN COLLEGE SUMO WRESTLERS: A PRELIMINARY STUDY}

\author{
Shigeyuki Nakaji', Kazuo Saito ${ }^{2}$, Junichito Kubota ${ }^{3}$, Toshio Ohshiro ${ }^{4}$ \\ 1: Department of Social Medicine, Hirosaki University School of Medicine, Hirosaki, Aomori, Japan; \\ 2: Nippon Sport Science University, Tokyo; 3: Kubota Junichiro Clinic, Tokyo; and \\ 4: Japan Medical Laser Laboratory, Tokyo
}

Sumo is a very powerful and competitive contact sport. Due to the extremely competitive nature of the sport and the required training, injuries are common both during training and the actual competition. Long-term lay-up of the competitor has to be avoided in order to maintain the level of muscle tone and mental concentration generated by the grueling training, so postinjury recovery time is kept to a minimum. A noninvasive therapy is therefore required, and the recent interest in the successful application of low level laser therapy (LLLT) in pain attenuation for a large number of pain types suggested that it might offer a new tool for sumo-related injuries. The current trial, with ten sports university sumo wrestlers, examined the effect of LLLT on injuries of the knee (five subjects) and foot (five subjects), using laser speckle flowmetry to assess the possible increase in superficial blood flow which has been associated with both pain attenuation and accelerated wound healing. An $830 \mathrm{~nm}$ $60 \mathrm{mWGaAlAs}$ diode LLLT system was applied on one point for $60 \mathrm{sec}$, and laser speckle flowmetry was performed before, during, immediately after, at $30 \mathrm{~min}$ and $60 \mathrm{~min}$ after irradiation. Decreased blood flow was seen intrairradiation, but an increase, significant in 7 of the 10 subjects was seen immediately postirradiation. This was maintained at significantly elevated levels in 4 subjects, while the remaining six decreased slightly, but in all ten subjects elevated levels of superficial blood flow were seen at one hour postirradiation, compared with preirradiation.

\section{S-8-4}

\section{THE TREATMENT OF IMPOTENCE RELATED WITH PROSTATE INFLAMMATIONS BY} MEANS OF INTRAVENOUS AND LOCAL NON INVASIVE LASER THERAPY

\author{
Hayk S. Arakelyan 1, 2, Norik Ch. Saribekyan 2 \\ Panarmenian Association of Laser Therapy and Integrative Medicine, \\ University of Traditional Medicine
}

To compare therapeutic efficiency of various forms of Laser Therapy - Intravenous, Local Non Invasive and their combination during impotence related with prostate inflammations. 26 male patients with the diagnosis of impotence related with prostate inflammations have been classified into the following 3 groups: 1.9 patients have been treated with combination of IV HeNe LBI and Local Non Invasive Laser Therapy (Group 1); 2.8 patients have been treated with IV HeNe LBI (Group 2) 3.9 patients have been treated with Local Non Invasive Laser Therapy on Prostate projection zone (Group 3). The patients' mean ages are 17-31. IV HeNe $(630 \mathrm{~nm}) \mathrm{LBI}$ has been done outputing power at the end of the light-guide inserted into a vein from 1 up to $3 \mathrm{~mW}$, exposition from $10-15$ minutes. Procedures were conducted on a daily base, 10 sessions on a course periodically - 20-25 days intervals; 3 courses in general. Local Non Invasive Laser Therapy has been given utilizing HeNe Laser on Prostate projection zone at intervals 5-8 minutes; on a course of 10-12 procedures, 3 courses in general. After treatment course the highest efficiency is noticed during combined laser therapy (Group 1) - in the patients of this group has been noticed as the improvement of potency as well as symptoms associated with prostate inflammations. In the second and third groups is noticed the improvement of the mentioned symptoms as well but the efficiency of these two groups is almost equal. 
S-9-1

\title{
LLLT IN WOUND HEALING AND MALE INFERTILITY \\ Peter Hasan
}

1.Laser Clinic, Dept. of Surgery, Pluit Hospital, Jakarta, Indonesia.; 2. Laser Clinic. Dept. of Surgery, Husada Hosp., Jakarta, Indonesia; and 3. Bakti Sinar Pratama Clinic, Jakarta, Indonesia. Since the report of Endre Mester on the Biostimulative Effects of Laser in 1967, LLLT was applied for plenty of indications in various medical fields. Our study in rabbits (1985) showed faster wound healing and better quality of scar in the laser group. To analyze the result of LLLT treatment, a retrospective review was performed on the 15.626 cases treated with LLLT over the period of 20 years (May 1985-June 2005). The overall good response rate was seen $63.87 \%$ of the case $(10062 / 15626)$, the best response was seen in Wound Healing Promotion group (92.05 \% - 6507/7069 cases), and where as good response in pain relief group were seen in only $63.87 \%$ (1478/2314 cases). In the 138 male infertility cases, the azoospermic patients ( 14 cases) showed no improvement at all in their sperm count after LLLT irradiation. In the severely and moderately oligospermic subjects (124 cases), a 2- to 3 -fold increase in sperm count was noted after 10 sessions of LLLT but only 11 had carry home babies after the treatment. In this series minor side effects were observed in only 0.12 $\%$ of the cases $(18 / 15626)$.

S-9-2

\author{
FERTILIZATION TREATMENT WITH LLLT \\ Dr. H. Iwahata, S. Endo, Y. Hirai \\ Iwahata Clinic, Shizuoka, Japan
}

From March to December, 2005, we had underwent fertilization treatments combined with low reactive level laser therapy (LLLT) for our patients with intractable infertility; we used in vitro fertilization, intracytoplasmic sperm injection (ICSI) and artificial insemination with husband's semen (AIH), together with LLLT. As a result of the treatments for 24 patients, 8 patients became pregnant (33.3\%) including 3 patients of abortion (37.5\%). The breakdown of the result is; ICSI : 19 patients with 7 of pregnancy, AIH: 4 patients/ 0 of pregnancy, and natural pregnancy: 1 patients/ 1 of pregnancy. As for AIH and natural pregnancy, the efficacy of LLLT was ill-defined due to the lack of cases. Under this situation, we noticed the efficacy of laser in ICSI. In 2005, 40 patients received ICSI in our clinic and 11 (27.5\%) of them got pregnant. 19 patients out of 40 underwent LLLT and others did not. As a result, 7 patients with LLLT and 4 patients without LLLT got pregnant. We surveyed the fertilization rate of the patients with and without LLLT, and found a noticeable difference between them. The former one (36.8\%) is higher than the latter one (19.0\%). Because of the rarity of the cases, it cannot be said that this clinical outcome is mainly due to LLLT with certainty, but it is possible that LLLT can improve the quality of ova, which leads to the pregnancy. It is hoped that the combination of conventional ART and LLLT improve the fertilization rate.

\section{S-9-3}

\section{TEN YEAR EXPERIENCE OF APPLYING LOW REACTIVE LEVEL LASER THERAPY AS AN ADJUNCTIVE TREATMENT FOR FEMALE INFERTILITY Shunji Fujii ${ }^{1,}$ Toshio Ohshiro ${ }^{2}$, Takafumi Ohshiro ${ }^{1,}$ Katsumi Sasaki ${ }^{1}$ \\ 1: Ohshiro Clinic, Shinanomachi, Tokyo, Japan; and 2: Japan Medical Laser Laboratory, Shinanomachi, Tokyo, Japan}

In Japan, the growing number of working women has caused the average age of marriage to rise, and also has increased the number of old age first time pregnancies. The average number of children that a single woman will bear in her lifetime has declined to a point where the population will decrease in the next few decades. The importance of treatment of infertility is growing and the result is of grave socio-economic consequences. Since October 1996, we 
have performed LLLT as an adjunctive treatment to 469 female patients being treated for infertility either by artificial insemination or by other assisted reproductive treatments (ART). The average age of the patients was 39.01years old, the average period of treatment prior to laser treatment was 5.29 years. The average number of ART was 9.56 times. Two types of lasers; mainly the $830 \mathrm{~nm}$, GaAlAs diode lasers and the $632.8 \mathrm{~nm}$, helium-neon laser and were used. An average of 12.37LLLT sessions was performed on the patients resulting in 99 confirmed pregnancies and 64live births from 57 patients. We believe that LLLT increases the peripheral blood flow to the ovary, raising the quality ovum and resulting in more pregnancies in an average age group of women where the expected pregnancy rate would be as low as 5 to $10 \%$. We report our ten year experience of treating infertile women with LLLT.

S-10-1

\section{LASER SAFETY: BEYOND SIGNS AND GOGGLES \\ Submitted by: Penny J. Smalley, R.N., CMLSO, MACORN \\ Technology Concepts International, Chicago, Illinois, USA}

LASER, OHS, IEC, NOHA, OD, MPE, AEL, PD, LSO, LSC, HCLS, HCP, LGAC, LCA, CW, PRR, VLT, SHOULDS AND SHALLS.........

A universe of laser standards, terminology, regulations, guidelines, professional recommended practices, policies, and procedures, governs the use of Class $3 \mathrm{~b}$ and Class 4 medical lasers, in many other countries around the world. Though we, as medical and nursing professionals, always strive to provide excellence in patient care, and a safe working environment for ourselves and our colleagues, we often feel confused as to how to read, interpret, and implement those rules that define our practice as we incorporate more and more laser equipment into daily use. An alarming result is a growing number of healthcare professionals becoming involved in accidents, incidents, and medical-legal situations. They are often unable to substantiate their practices with evidence based best practice documents for safety, because of lack of educational resources, and guidance. This session will examine international regulations and standards, offering useful practice recommendations, based on a risk management perspective, with a focus on administrative, and procedural control measures. At the conclusion of this session, participants will be able to:

1. Discuss the scientific basis for laser safety, as a means for risk assessment, and development of control measures.

2. Discuss elements of the international standards, as a basis for operational policies and procedures in healthcare practice settings, in both hospital and office practice environments.

3. Discuss the use of audit as a quality assurance tool for mandating compliance and safe practice.

\section{S-10-2}

\section{ISO 9001:2000 STANDARD CERTIFICATION - A MUST FOR ALL MODERN PRIVATE LASER CLINICS Premysl Fryda}

European Medical Laser Association (EMLA), Prague, Czech Republic

International standards ISO 9001:2000 has been first introduced by industry manufacturers in order to stress quality of their products. Later on, these standards spread to providers of services and became generally known as a guarantee of a certain level of quality. And quite recently, large hospitals as well as little private clinics have started introducing these quality standards as well, though adjusted to specific conditions in providing medical care. The main purpose of this paper is to introduce the main benefits of certification of management of quality. Laser therapy and laser surgery mean medicine based on using high-tech devices, strictly limited by applicable hygienic and safety rules. Working with top technology should automatically include working under top quality standards. Certification of ISO 9001:2000 
standards being applied to a private clinic or medical practice brings the following benefits: $\checkmark$ inventory of all procedures being carried out in the course of your everyday work, identification of weak points and elimination of potential defects within your system,

$\checkmark$ improved certainty for patients in terms of quality and safety of medical care provided,

$\checkmark$ more guarantees for public and private health care insurance providers,

$\checkmark$ safer background for you in cases of potentially threatening forensic audits and patients' lawsuits.

And, apart from that, don't forget that ISO 9001:2000 certification is also a matter of prestige for both your patients and colleagues!

\section{S-10-3}

TRANSCUTANEOUS PHOTOMODIFICATION OF BLOOD COMPONENTS AS A PRINCIPAL MECHANISM OF ANTI-INFLAMMATORY, IMMUNOMODULATORY AND WOUND HEALING STIMULATORY EFFECTS OF LIGHT

\section{K.A. Samoilova, N.A. Zhevago}

Institute of Cytology, Russian Academy of Sciences, St. Petersburg, Russia

In the 1980s, intravascular and extracorporeal laser irradiation of small amounts of patient's own blood was developed in Russia and since that time successfully used for treatment of inflammatory, cardio-vascular, infectious, skin diseases and severe wounds. This suggests that photomodification of blood components plays the key role in light therapy. In the case of percutaneous application, the visible and IR light penetrating the skin can directly affect the blood circulating in the superficial dense network at a relatively low rate. In our randomized, placebo controlled, double blind trials, a small area of volunteers' body surface was exposed to polychromatic polarized light $\left(480-3400 \mathrm{~nm}, 95 \%\right.$ of polarization, $\left.12 \mathrm{~J} / \mathrm{cm}^{2}\right)$ and in parallel blood samples of the same subjects were irradiated in vitro. At least 6 groups of effects have been revealed at 0.5-24 h post-irradiation: Improvement of the rheological, transport, oxygen-transport properties of red blood cells and microcirculation (1); A decrease in platelet aggregation and upregulation of anticoagulation system (2); Activation of functional state of all types of leukocytes (3); A dramatic decrease in the plasma content of pro-inflammatory cytokines (TNF-a, IL-6, IL-12, IFN-g) in subjects with their high pre-values and an increase of anti-inflammatory cytokines concentration (IL-10, TGF-b1) as well as of IFN-g in individuals with its low and normal values (4); Enhancement of the growth promoting activity of blood plasma for human keratinocytes, endotheliocytes, and fibroblasts, which develops in parallel with increase in plasma content of some growth factors - PDGF-AB, TGF-b1 (5); Activation of DNA-repair system in injured target cells by soluble factors of photomodified autologous blood (6). These changes develop at contact of the circulating blood with a small amount of transcutaneously photomodified blood that is able to transfer the light-induced effects to unirradiated blood. 